\title{
Grazing incidence scattering
}

\author{
Max Wolff* \\ Department for Physics and Astronomy, Uppsala University, 75120 Uppsala, Sweden
}

\begin{abstract}
Reflectometry experiments probe the scattering length density along the normal of interfaces by analysing the specularly scattered intensity. Lateral fluctuations result in intensity scattered away from the specular condition. In this paper the principles and peculiarities of grazing incidence scattering experiments are explained. One specific example, the self assembly of polymer micelles close to interfaces, is taken as a show case in order to introduce the scattering geometry and accessible length scales. The basic idea of the distorted wave Born approximation is lined out and some scientific examples are summarized.
\end{abstract}

\section{The importance of surface effects}

With the development and miniaturization of modern technology the need for advanced experimental methods in surface science has increased tremendously. This fact is reflected, for example, by the development of atomic probes [1] or electron microscopy [2]. In the field of x-ray and neutron scattering the number of reflectometers, which are most relevant for surface science, is growing and with the years specular reflectometry measurements have become a routine tool to extract the density profiles of materials across interfaces with almost atomic resolution (see chapter 04001 by F. Cousin and A. Chenneviere). A list of neutron reflectometers as well as relevant citations describing those can be found in Ref. [3] and an introduction in reflectometry and grazing incidence is found in [4]. However, specular reflectivity measurements can only reveal the scattering length density (SLD) profile across an interface with the scattering potential averaged in the plane of the interface and over the coherence volume of the beam. To become more specific, inter-diffusion and roughness or even regular patterns on a surface can not be characterized (see Figure 1). In the case of roughness lateral correlations in the plane along the interface may be present. These give rise to interferences and a modulation of the scattered intensity. The lateral correlations can be extracted from so called offspecular (OFFSpec), grazing incidence small angle scattering (GISAS) or diffraction (GID). During the last 15 years most neutron reflectometers have become equipped with position sensitive detectors allowing an efficient collection of OFFSpec and grazing incidence scattering (GIS) data. Still the method is not exploited to its full potential, which results on the one hand from the fact that easy to use and robust fitting routines are not yet developed and on the other hand from the strongly limited flux and dynamic range on neutron instruments. Typically, OFFSpec and GIS intensities are three or more orders of magnitude lower in intensity than their specular counterparts.

^e-mail: max.wolff@physics.uu.se 


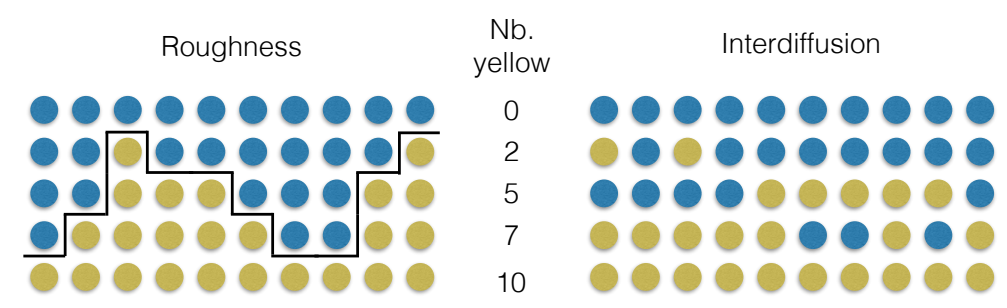

Figure 1. Roughness and inter-diffusion: In the left panel a continuous line can be drawn between the two materials, whereas in the right panel isolated islands exist. The integrated number of yellow and blue circles along the horizontal direction is the same. Specular reflectivity can not distinguish between the two scenarios.

This manuscript is structured as follows: First the scattering geometry and the peculiarities of OFFSpec and GIS is explained on a specific example of polymer micelles self assembling at a solid boundary. Then the distorted wave Born approximation (DWBA) is outlined and finally some scientific examples are presented.

\subsection{Scattering geometry}

The scattering geometry in neutron reflectometry (NR) is usually defined in cartesian coordinates with the $\mathrm{z}$ direction along the normal of the interfaces. The $\mathrm{x}$-direction is in the scattering plane and $\mathrm{y}$ is perpendicular to $\mathrm{x}$ and $\mathrm{z}$ (see Figure 2). In the case of a buried liquid interface a neutron beam may pass trough a silicon wafer, get scattered at the solid-liquid boundary and detected by an area detector. Note, since the absorption of neutrons in silicon is low and the scattering potential of deuterated liquids is large, such experiments can be performed in a straight forward way with a minimum loss in flux and a critical momentum transfer of total external reflection present.

The intensity scattered at the same incident and exit angles (orange arrows in Figure 2) is the specular reflectivity and contains the information about the SLD profile along the surface normal. Intensity scattered at different exit angles but still in the scattering plane (symbolized green) is usually called OFFSpec and intensity registered for exit angles out of the scattering plane is small angle scattering (SAS). The three components of the scattering vector $\mathrm{Q}$, along the $\mathrm{x}, \mathrm{y}$ and $\mathrm{z}$-direction, are calculated

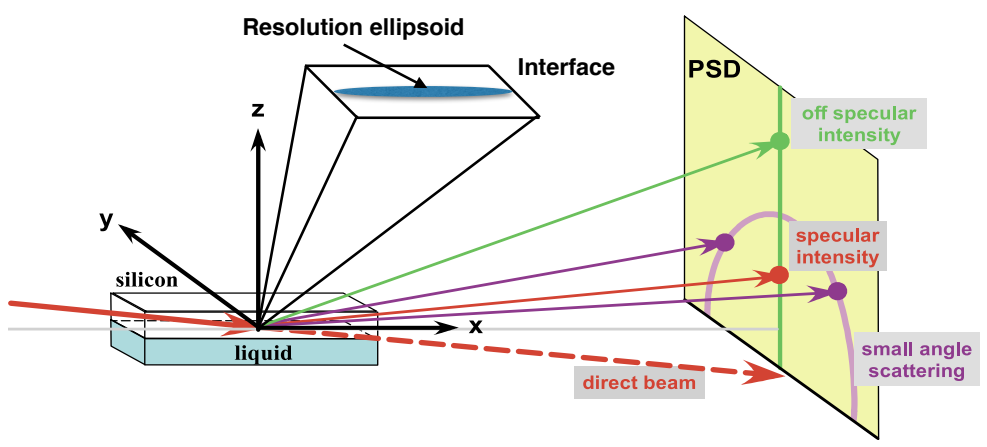

Figure 2. Scattering geometry for GIS experiments from the solid-liquid boundary [5]. 


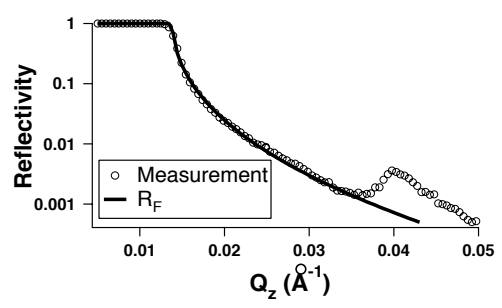

Figure 3. Reflectivity data taken with a micellar polymer solution in the liquid phase in contact with a silicon wafer compared to the Fresnel-reflectivity $R_{F}$.

as follows:

$$
\begin{aligned}
Q_{x} & =\frac{4 \pi}{\lambda}\left(\cos \alpha_{f} \cos \phi-\cos \alpha_{i}\right) \\
Q_{y} & =\frac{4 \pi}{\lambda} \sin \phi \\
Q_{z} & =\frac{4 \pi}{\lambda}\left(\sin \alpha_{i}+\sin \alpha_{f}\right)
\end{aligned}
$$

with $\alpha_{i}$ and $\alpha_{f}$ the angle of the incident and exiting beam with respect to the sample's surface, $\lambda$ the wavelength and $\phi$ the scattering angle out of the scattering plane.

\section{Block-polymers and micelles}

In the following the peculiarities of grazing incidence small angle neutron scattering (GISANS) and OFFSpec will be explained on one specific example, namely aqueous solutions of block polymers and their assembly at the solid-liquid boundary. This system offers a strong scattering signal on all length scales relevant for GIS experiments. In aqueous solution the molecules tend to form micelles (self-aggregartions of molecules). Depending on temperature and volume fraction these may form liquid or crystalline phases. The sample is a $18.5 \%$ (in weight) solution of Pluronic F127 $\left(\mathrm{EO}_{99}-\mathrm{PO}_{65}-\mathrm{EO}_{99}\right)$ in deuterated water providing a good contrast for neutrons. The bulk properties of such samples are known in great detail [6,7]. In the case of F127 face centred cubic (fcc) crystals are formed at elevated concentrations or temperatures.

Figure 3 shows the specular reflected intensity taken with the sample in a liquid phase at $295 \mathrm{~K}$ compared to the Fresnel-reflectivity. The data is corrected for the SAS by substracting the intensity for a detector off-set angle, from the specular condition, of $0.3^{\circ}$. At $Q_{z}=0.04 \AA$, the intensity is increased. This Bragg peak indicates several layers of adsorbed micelles at conditions, which are still in the liquid phase with no fcc structure present in the bulk.

\section{Off-specular and GISANS}

In the crystalline phase (temperature $298 \mathrm{~K}$ ) a fcc dense packed structure is formed. Figure 4 (left panel) represents a GISANS pattern collected on an area detector for a monochromatic incident beam impinging the sample under an incident angle $\alpha_{i}$ close to the critical angle of total external reflection $\alpha_{c}$. Specifically, $\alpha_{i}=0.30^{\circ}$ results in a penetration depth into the liquid of $40 \mu \mathrm{m}$ (extinction length 

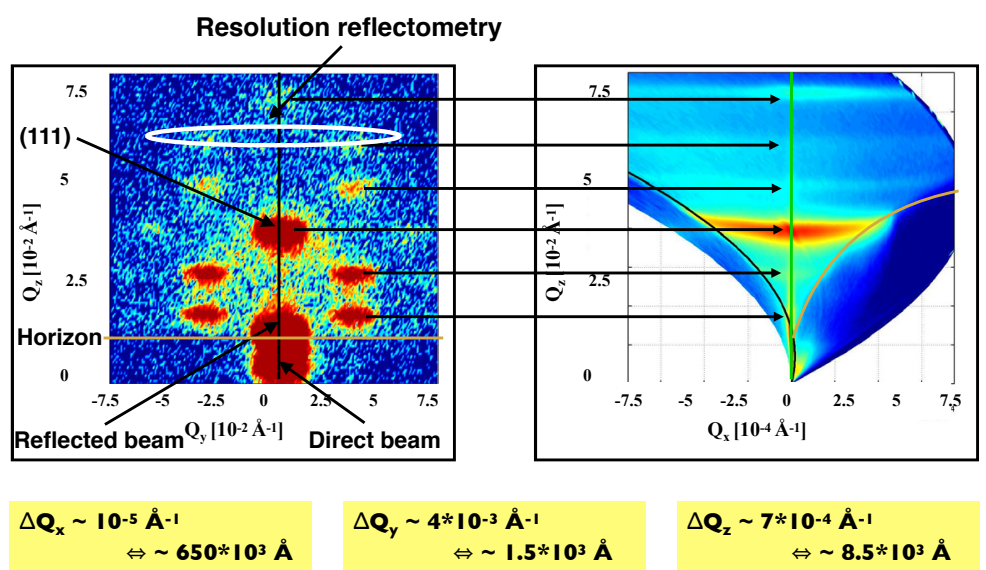

Figure 4. Scattering patterns for a micellar crystal in contact to a silicon wafer. The panel on the left hand side depicts the GISANS pattern form the cubic close packed structure. The panel on the right hand side depicts reflectometry data (specular and OFFSpec) from the same sample but with a relaxed resolution along the ydirection [5].

for a drop of 1/e of the incident intensity). $\alpha_{c}$ for a neutron wavelength of $0.44 \mathrm{~nm}$ is $0.25^{\circ}$ and defined by the SLD of silicon and the polymer solution. For no momentum transfer $Q=0$ the direct beam hits the detector at the very bottom in the middle. Slightly above the reflected beam is visible. Half way between these two peaks the sample horizon (brown line), dividing intensity transmitted by and reflected at the interface, is found. Clearly below the horizon the intensity is low (dark blue region below $\mathrm{Q}_{z}=0.01 \AA^{-1}$ ) due to the enhanced absorption or incoherent scattering inside of the liquid sample as well as of the sealing of the liquid cell [10]. Note: the image shows raw data and $Q_{z}$ was not corrected for refraction effects $Q_{z, \text { in }}=\sqrt{Q_{z}^{2}-Q_{c}^{2}}$, with $Q_{z \text {,in }}$ the $Q_{z}$ value inside the liquid. All other reflections are Bragg peaks resulting from a cubic dense packing of the polymer micelles close to the solid interface [11]. The projection of the resolution on the $\mathrm{x}, \mathrm{y}$ and $\mathrm{z}$ axis for GIS is dependent on $\alpha_{i}$ and the exit angle $\alpha_{f}$, since the coordinate system is fixed to the sample and not to the instrument [5]. Specifically, for this example and $Q=0.04 \AA^{-1}$ the resolution $Q_{x}=10^{-5} \AA^{-1}$ is determined by the pixel size of the detector and the scattering angle. The resolution $Q_{y}=4 \times 10^{-3} \AA^{-1}$ and $Q_{z}=7 \times 10^{-4} \AA^{-1}$ is given by horizontal and vertical slits. In real space the resolution is defined by the coherence volume of the beam projected onto the respective axis of the coordinate system and is roughly $65 \mu \mathrm{m}, 150 \mathrm{~nm}$ and $850 \mathrm{~nm}$ along the $\mathrm{x}$, y and z-direction, respectively, for the incident beam angle of $0.3^{\circ}$.

On the monochromatic instrument $\alpha_{i}$ and $\alpha_{f}$ are changed and for each setting and an image, similar to the one shown in the panel on the left hand side, is detected. The panel on the right hand side (Fig. 4) shows the result of such a NR measurement, including the OFFSpec scattering. In this representation the horizon is again represented by a brown line. The two panels on the left and right (Figure 4) are orthogonal but intersect along the black lines. This line is almost identical with the sample horizon in the right panel. Due to the non-linear transformation of the coordinate $Q_{x}$ the black line is curved in the panel on the right hand side.

For the NR measurement the resolution along $y$ has been relaxed to $0.15 \AA^{-1}$ (see white resolution ellipsoid in Fig. 4) in order to gain in flux. As a result a contribution from the Bragg reflections with 

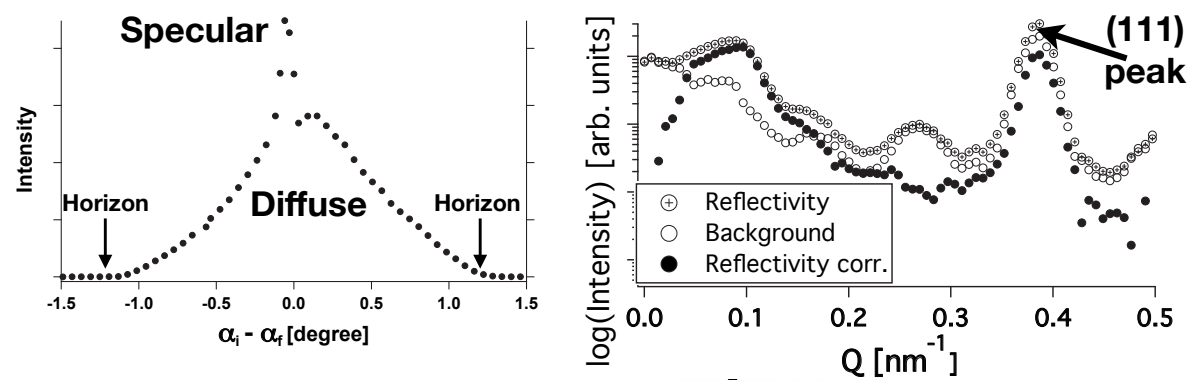

Figure 5. Left panel: Rocking scan for the sample in the crystalline phase at $\mathrm{Q}_{z}=0.38 \mathrm{~nm}^{-1}$. Right panel: Correction of the specular scattered intensity for SAS.

$Q_{y} \neq 0$ is detected in the $\mathrm{Q}_{x}, \mathrm{Q}_{z}$ plane. In order to extract the true specular reflectivity this contribution has to be corrected for, since the intensity is not scattered specular but appears in the specular direction. This is a severe challenge for samples with strong SAS, when the SAS can be equal or stronger than the specular signal. Figure 5, right panel, demonstrates this effect [20]. The detected specular NR is plotted on a logarithmic scale versus $Q_{z}$ (circles marked with a cross). The background, for an offset of $\alpha_{i}$ and $\alpha_{f}$ of $0.25^{\circ}$, is represented by open circles. For $Q_{z}>0.2 \mathrm{~nm}^{-1}$ the reflectivity is dominated by SAS and the true specular signal (black dots) can hardly be extracted with statistical significance.

The panels on the right and left hand side, Fig. 6, illustrate the unit cell of the cubic close packing and the GISANS plotted as color map onto the Ewald sphere, respectively. The detector plane represents a curved surface with $\mathrm{Q}=0$ at the position of the direct beam. The second and only other point where the in-plane coordinates $Q_{x}$ and $Q_{y}$ are zero is the point where the specular scattered intensity is detected. All other points on the detector have none-zero $Q_{x}$ and/or $Q_{y}$ components.

Considering a densely packed cubic structure at the interface, the (111) lattice planes are parallel to the solid-liquid boundary and the Bragg reflections resulting from them should have no in-plane component $\left(Q_{x}=Q_{y}=0\right)$. This implies that the (111) reflection can only be detected in a GISANS
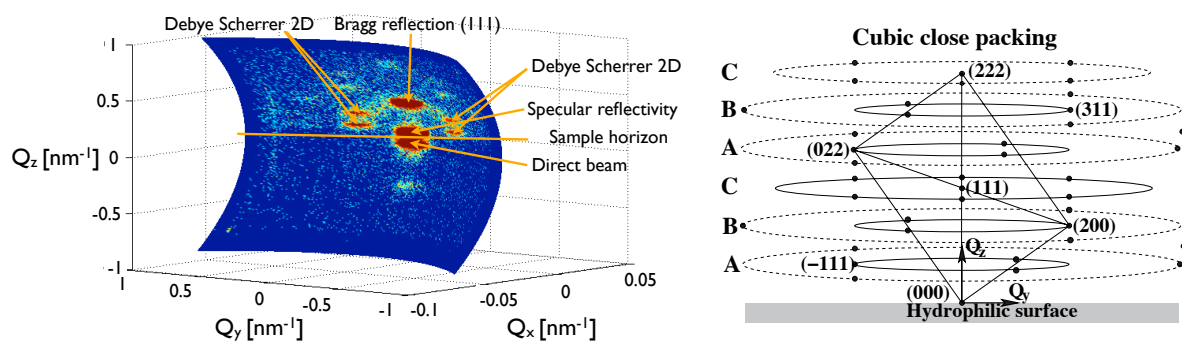

Figure 6. Left panel: GISANS scattering pattern plotted on the surface of the Ewald sphere in three dimensions [18]. Right panel: Reciprocal lattice for a cubic close packing (ccp). The dots mark the positions of reciprocal lattice points. For a two-dimensional powder with the unique axis along $Q_{z}$ perpendicular to the interface these points are smeared out into circles. 


\section{Correlated roughness}
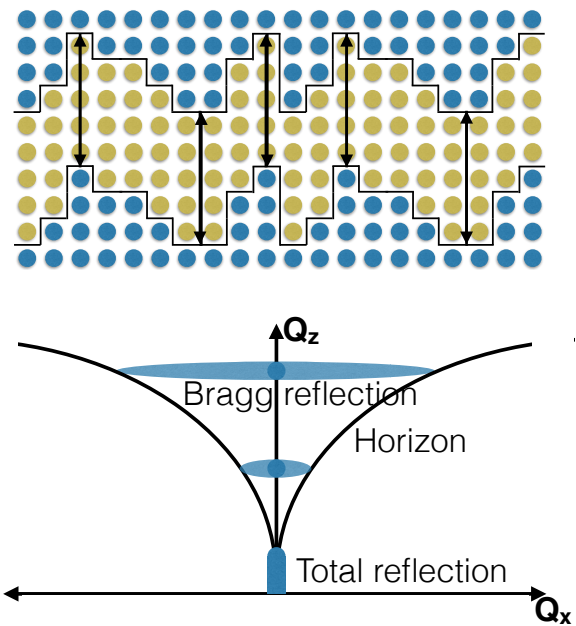

Uncorrelated roughness
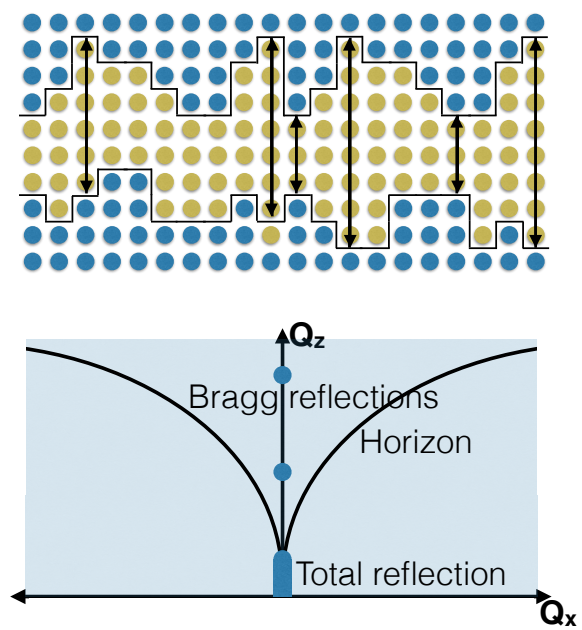

Figure 7. Structure and off-specular scattering from rough surfaces for perfectly correlated (left panels) and uncorrelated (right panels) interfaces.

experiment if it has a certain rocking width along $Q_{x}$ or in other words correlated roughness of the micellar layers is present. All other four Bragg reflections are only visible if the crystalline structure is a two dimensional powder with respect to the sample interface [5], since the $Q_{y}$ values are much larger than the $Q_{x}$ values and the probability of a crystal orientation with the Bragg reflection on the Ewald sphere is unlikely.

The (111) reflection at $Q_{z} \approx 0.38 \mathrm{~nm}^{-1}$ and $Q_{y}=0$ is smeared out in the reflectivity measurement (right panel) along $Q_{x}$ due to correlated roughness. Figure 5, left panel, represents a cut along this reflection along $\mathrm{Q}_{x}$. Such a cut is called Rocking curve, the $\alpha_{i}$ and $\alpha_{f}$ are scanned for given scattering angle (see $\mathrm{x}$-axis in Figure 5, left panel). The narrow component is the specular scattered intensity and reflects long range orientational correlations over the coherence volume of the neutron beam [21], indicated by the ellipsoid in Fig. 2 (resolution ellipsoid). The broad component is related to diffuse or OFFSpec scattering and results from the finite lateral correlations of the SLD fluctuations contributing to the Bragg peak along the plane of the interface. As mentioned the length scale probed along the $\mathrm{x}$-direction is about two orders of magnitude larger than the one probed along $\mathrm{y}$ and $\mathrm{z}$ (see Figure 4). The GISANS together with an analysis of the width of the (111) reflection in the off-specular scattering regime can be used to analyse the texture as well as the size of the micellar crystallites [16]. Figure 7, left and right panel, depicts the extreme cases of perfectly correlated and uncorrelated interface roughness, respectively, and the resulting OFFSpec scattering. For a perfectly correlated roughness (left panels) between layers the OFFSpec scattering becomes concentrated along so called Bragg sheets, showing up as horizontal lines at a certain $Q_{z}$ and running along $Q_{x}$. The width of the sheets is the reciprocal lateral correlation length. For uncorrelated layers the OFFSpec scattered intensity is smeared out over the whole Q space. Note, the discussion above is based on kinematic approximations and neglects the dynamic effects which can be important in reflectometry experiments. However, dynamical effects (apart from total reflection) are typically important for very well ordered samples, which are hardly found in soft matter applications. 


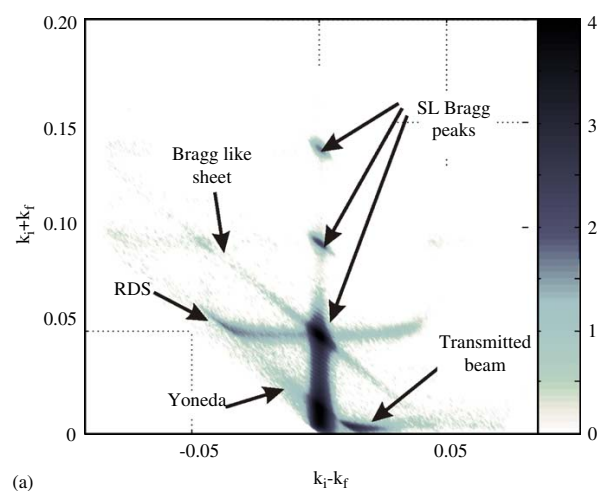

Figure 8. Two-dimensional intensity maps of the scattering from a $[\mathrm{Ti}(70 \AA) / \mathrm{Ni}(70 \AA)]_{20}$ multilayer plotted as function of $k_{i}+k_{f}$ and $k_{i}-k_{f}[12]$.

Still dynamic effects resulting in so called Yoneda and resonant diffuse scattering (RDS) will be discussed briefly below. For illustration Fig. 8 depicts a scattering pattern collected with a TiNi multilayer with 20 repetitions [12]. The intensity is plotted over $k_{i}+k_{f}$ and $k_{i}-k_{f}$ instead of $Q_{x}$ and $Q_{z}$ to avoid the compression of the scattering pattern close to the critical momentum transfer, resulting from the non-linear transformation to Q [13]. Yoneda scattering [14] results from an constructive interference of the incoming and outgoing wave field at the critical momentum transfer of total external reflection and enhances scattering from roughness along so called Yoneda wings (sample horizon). In addition, since the incident or exiting wave impinges under the critical momentum the scattering pattern gets distorted close to the horizon. This results in an upturn (towards larger $\mathrm{Q}_{z}$ values) of the Bragg sheets in this region. For multilayers there is additional interference of the waves scattered at each interface and again for constructive interference roughness scattering is enhanced resulting in RDS or Holy scattering [15].

\section{Modes of measurement}

As described in the previous chapters neutron scattering experiments can be done in two modes. The classical way are monochromatic instruments with a continuous flux of neutrons. For each point in $Q$ the scattering angle as well as the incident angle is chosen with respect to the sample, see Figure 9 , bottom panel. For the time of flight (TOF) method a pulsed neutron beam is used with a polychromatic beam and the wavelength is extracted from the time of flight of the neutron from the source to the detector. With this method a range of momentum transfers is measured for one given setting of the instrument, see Figure 9, top panel.

As a result a whole range of $\mathrm{Q}$ values around $\mathrm{Q}_{c}$ and a range of penetration depth from the boundary can be probed. For a momentum smaller than $\mathrm{Q}_{c}$ the penetration depth is small, on the order of 20 $\mathrm{nm}$ (see Figure 10). Figure 10 shows the penetration depth of the neutrons into the liquid calculated according to Ref. [8, 9], using the sum of the absorption and the incoherent scattering cross sections. Note, since incoherent scattering is isotropic it only adds to the background but contains no information relevant for GISANS or OFFSpec scattering. Still the neutrons scattered incoherently are "lost" and can as such be treated as the absorbed ones. At the critical momentum the penetration depth has a distinct jump, in the case presented here by about four orders of magnitude from some nm straight to 

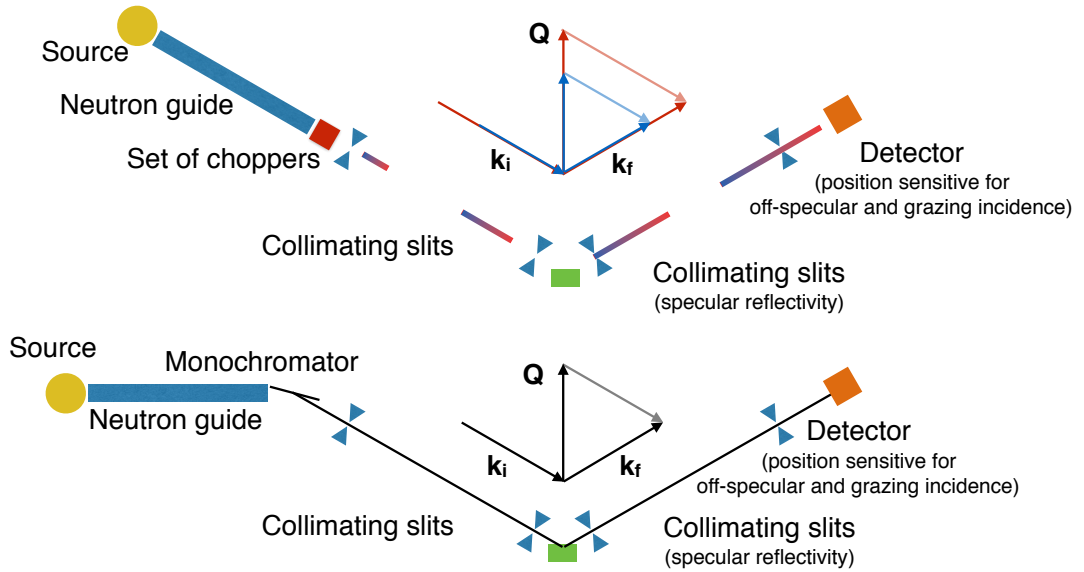

Figure 9. Modes for reflectometry experiments, the top panel shows time of flight or polychromatic instruments and the bottom panel monochromatic ones. On polychromatic instruments a range of $Q$ values is probed for one setting of the instrument.

micrometers, which is typical for neutrons. The height of the jump at the critical momentum is related to the extinction properties of the material. The curve shown in Figure 10 is calculated for the siliconpolymer solution interface discussed above. The incoherent scattering cross section is included in the absorption and extinction related to scattering is neglected. It is seen that the penetration depth into the liquid changes by four orders of magnitude within $10 \%$ above and below $Q_{c}$.

This implies that taking independent data in regions 1, 2 and 3 and achieving depth resolution is extremely challenging since an excellent $\frac{\Delta Q}{Q_{c}}$ is needed. In particular any tails of the resolution function

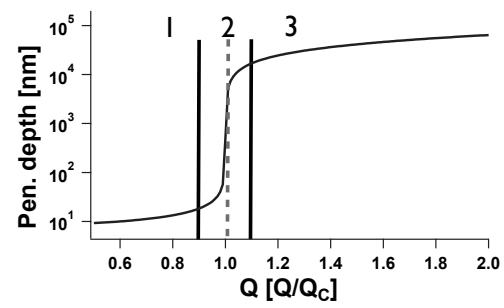

Figure 10. Penetration depth of a neutron into a micellar solution (20\% of protonated polymer solved in $80 \%$ deuterated water) being reflected at the silicon polymer interface. 
will immediately result in neutrons penetrating $\mu \mathrm{m}$ into the liquid and can dominate the scattering pattern [17]. $\frac{\Delta Q}{Q}$ is calculated by the following equation taking into account the wavelength distribution $\Delta \lambda$ and a angular divergence $\Delta \theta$ :

$$
\frac{\Delta Q}{Q}=\sqrt{\left(\frac{\Delta \lambda}{\lambda}\right)^{2}+(\Delta \theta \cot \theta)^{2}}
$$

For small angles $\theta$ this can be simplified:

$$
\frac{\Delta Q}{Q}=\sqrt{\left(\frac{\Delta \lambda}{\lambda}\right)^{2}+\left(\frac{\Delta \theta}{\theta}\right)^{2}}
$$

The most efficient use of neutrons is achieved for equal relative resolutions regarding wave length and divergence. For a time of flight instrument the wavelength resolution is given by the length of the instrument, the time resolution of the detector as well as by the neutron pulse length and for a monochromatic instrument by the mosaic of the monochromator and the divergence in the neutron guide or the specifications of the velocity selector. The divergence of the beam can be adjusted on both types of instruments by slits. Let us assume a wavelength and angular resolution of $1 \%$. Regarding the wavelength this can easily be reached with time of flight methods or monochromators. For the angular resolution at a critical angle of $0.2^{\circ}$ a $1 \%$ resolution implies a divergence of $0.002^{\circ}$ or two pinholes of $1 \mathrm{~mm}$ diameter at a distance of almost $30 \mathrm{~m}$. In addition the footprint of e.g. $0.175 \mathrm{~mm}$ for a $5 \mathrm{~cm}$ sample under the angle of $0.2^{\circ}$ is small.

Figure 11 depicts patterns of intensity measured on SANS-2D (Rutherford Institute, Didcot, England) for the polymer solution discussed above but now in contact to two silicon wafers with distinct surface energies [18]. The incident beam was collimated to provide a divergence of $0.29^{\circ}$ and $0.04^{\circ}$ along y and $\mathrm{z}$ direction, respectively. The incident beam angle was chosen to be $0.3^{\circ}$ resulting in a critical wavelength for total external reflection of $\lambda_{c}=5.4 \AA$.

The three pictures for the hydrophilic silicon surface treated with piranha (top panels) and the hydrophobic one coated with octadecyltrichlorosilane (OTS) (lower panels) were taken simultaneously for one incident beam angle with a fixed detector angle. The left panels correspond to the integration of the detector images for wavelengths ranging from 6-15.6 ̊ resulting in a penetration depth of the neutron beam into the polymer solution of approximately $10 \mathrm{~nm}$ (region 1 in Figure 10). The intensity integrated for short wavelengths of 1.75-5 $\AA$ and thus a large penetration depth of about $30 \mu \mathrm{m}$ is depicted in the right panels. The central column summarizes the intensities for wavelengths integrated around the critical wavelength, 5-6 $\AA$, resulting in an intermediate penetration depth of about $10 \mu \mathrm{m}$. For the smallest penetration depth of about $10 \mathrm{~nm}$ a ring of scattered intensity is visible around the direct beam $(\mathrm{Q}=0)$ for the hydorphobic substrate. This ring is absent for the micelles in contact with the hydrophilic surface. Note, the presence of the 111 reflection for these wavelength demonstrates the limited depth sensitivity of the measurement, since the constructive interference arises from layers separated by more than $10 \mathrm{~nm}$ [17]. The difference in the intensity distribution for the sample in contact to one or the other solid substrate becomes more pronounced for larger penetration depths (middle panels). At least ten Bragg reflections can be distinguished with the sample in contact to the hydrophilic surface, whereas still the ring of scattered intensity is dominant for the hydrohpobic one. For the largest penetration depth (right panels) both detector images are almost identical, reflecting the bulk structure and a vanishing influence of the interface.

Another example of depth resolved time-of-flight GISANS addresses the morphology of defect rich deuterated poly(styrene-block-methyl methacrylate), P(S-b-MMAd), diblock copolymer films [19]. 


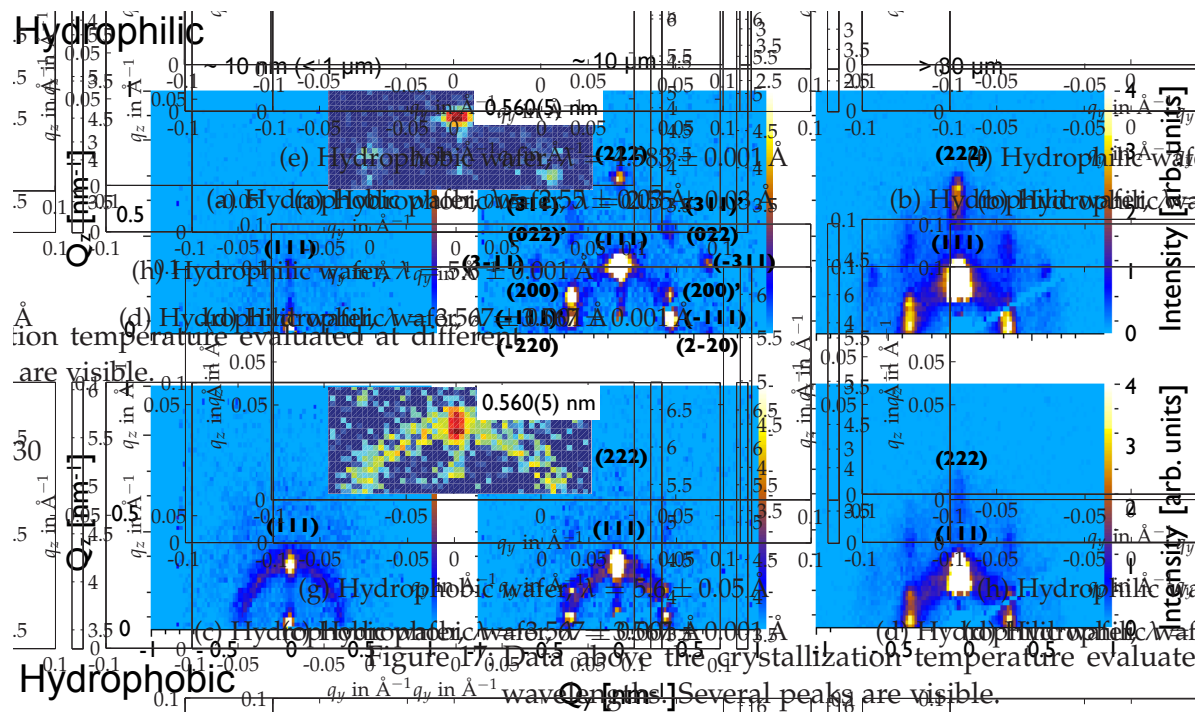

Figure 11. GISANS data taken for a solution of the polymer F127 in deuterated water in contact with a silicon surface treated with piranha (hydrophilic, top panels) and OTS (hydrophobic, lower panels). The penetration depths increases from left to right due to the different wavelengths in the incoming beam.

\section{Reflection, refraction and scattering}

Often the distorted wave Born approximation (DWBA) [22] is applied to describe OFFSpec and GIS. This method is a first order perturbation theory and requires that the SLD profile along the surface normal is known. This allows the calculation of the wave amplitude inside the sample. The scattering cross sections are then weighted with this wave amplitude in order to quantify the amount of OFFSpec and SAS. Figure 12 depicts the principle of the DWBA for a single interface. First the problem for a perfectly flat interfaces (plane waves) is solved and then the in-plane correlations are added as small perturbation (spherical waves). Luckily, the one-dimensional problem can be solved exactly but requires a specular reflectivity measurement. From that the SLD profile averaged over the coherence volume of the beam and the wave amplitude at different depth can be extracted. Note, since the coherence of the beam is strongly anisotropic it might be necessary to distinguish between a coherent (sum of wave amplitudes) and an incoherent (sum of intensities) summation along the $Q_{x}$ and $Q_{y}$ directions. This is particularly important for anisotropic samples, like e.g. stripe patterns [25] (see Fig. 13). The transmitted intensity below the critical momentum transfer is purely imaginary and becomes:

$$
|\Psi(z)|^{2}=t_{r}^{2} e^{-\frac{z}{l}}
$$

At each interface the neutron wave function has to be continuous and can be written as follows:

$$
\begin{gathered}
\Psi(z)=e^{i k_{i} z}+r_{F} e^{-i k_{i} z} \\
\Psi(z)=t_{r} e^{-i k_{r} z}
\end{gathered}
$$

Equation 7 and 8 describe the wave function outside and inside the sample, respectively. $r_{F}$ and $t_{r}$ denoted the Fresnel and transmission coefficients and $k_{i}$ and $k_{r}$ the momentum of the incident and 


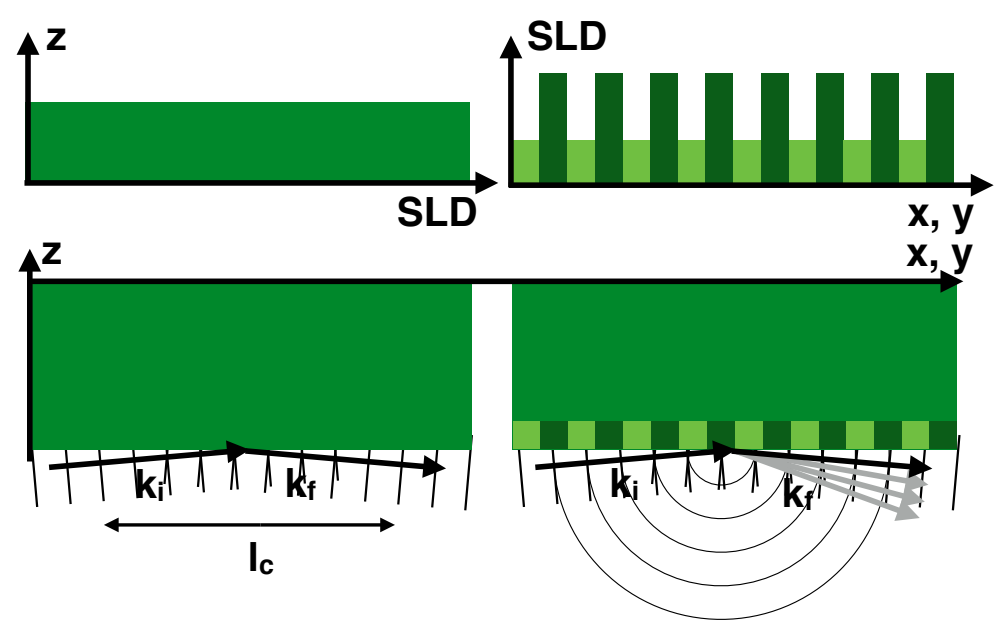

Figure 12. Scattering from lateral structures: The panels on the right hand side depict the scattering of plane waves from a sharp interface. On the left hand side lateral correlations are introduced. These give rise to spherical waves and OFFSpec scattering.

refracted wave. The intensity is calculated from the square of the wave function:

$$
|\Psi(z)|^{2}=1+r_{F}^{2}+2 r_{F} \cos \left(k_{i} z\right)
$$

The wave field oscillates as a function of $z$ and forms a standing wave, with amplitude two, at the critical momentum transfer. This enhancement is called Yoneda scattering. Note, that this effect is similar to the enhancement of the wave field at Bragg reflections (constructive interference) leading to RDS for correlated roughness [15]. The enhancement of the wave field at the surface can be amplified further by using resonator layers [23, 24].

Once knowing the incident and outgoing wave fields for the mean potential the scattering cross section for off-specular or GIS can be calculated from lateral fluctuations $\triangle S L D=S L D-\langle S L D\rangle$.

$$
\frac{d \sigma}{d \Omega}=\left\langle\left|f\left(\vec{q}_{\|}, p_{0 f}, p_{0 i}\right)\right|^{2}\right\rangle
$$

with

$$
f\left(\vec{q} \|_{\|}, p_{0 f}, p_{0 i}\right)=-\int d \vec{r} \Psi_{f}(\vec{r}) \Delta S L D(\vec{r}) \Psi_{i}(\vec{r})
$$

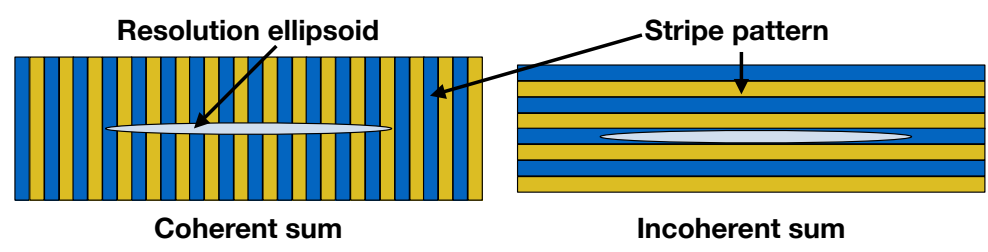

Figure 13. Coherent and incoherent summation: Depending on the orientation of the resolution ellipsoid with respect to in-plane correlations a coherent (left panel) or incoherent (right panel) summation has to be applied. 
Using the fact that the normal and lateral components of the wave function are orthogonal and the components factorize we get the result for the scattering cross section:

$$
\frac{d \sigma}{d \Omega}=\left\langle\left|f\left(\vec{q}_{\|}, p_{0 f}, p_{0 i}\right)\right|^{2}\right\rangle
$$

with:

$$
f\left(\overrightarrow{q_{\|}}, p_{0 f}, p_{0 i}\right)=-\int d z \Psi_{f}(z) \Delta S L D\left(q_{\|}, z\right) \Psi_{i}(z)
$$

and:

$$
\Delta S L D\left(\overrightarrow{q_{\|}}, z\right)=\int d \overrightarrow{r_{\|}} \Delta S L D\left(\overrightarrow{r_{\|}}, z\right) e^{i \overrightarrow{q_{\|}} \overrightarrow{r_{\|}}}
$$

This result is a Fourier transform as normally received in the Born approximation but weighted with the incident and outgoing wave amplitude. The formalism of the DWBA as well as examples from magnetic thin films and laterally patterned structures are summarized in Ref. [26]. While there is an agreement among software packages and fitting tools for specular reflectivity, currently programs to calculate diffuse scattering as well as GIS are sparse, e.g. the project BornAgain [27] allows the simulation of GISANS scattering patterns.

Since the DWBA is a first order perturbation theory the condition that the lateral fluctuations have to be small compared to the changes in SLD along the surface normal has to be fulfilled. For many experiments this may not be the case. As an example in the data set presented in Figure 3 right panel, the SAS intensity is almost equal to the reflected intensity. Concerning this fact a quantitative evaluation of GIS data is challenging and many studies restrict themselves to extract characteristic length scales or relative peak intensities.

\section{Examples}

The number studies OFFSpec and/or GIS using neutrons is limited. This fact results from the relatively low brilliance of the current neutron sources. Synchrotron x-ray sources offer orders of magnitude higher brilliance and GIS techniques are used there more routinely [28, 29].

Neutrons, due to their spin, high penetration power and isotope sensitivity, offer unique possibilities for the study of magnetism, buried interfaces and soft matter. In the case of GIS from soft matter, in particular buried interfaces, like the solid-liquid boundary discussed above are of high interest as well as the self organization and dewetting of polymers on solid substrates. Following along this line, in the end of the 80's, Anastasiadis et al. [30] demonstrated that flat multilayers of poly(styreneb-deuterated-methylmethacrylate) are formed on a substrate. In the study they used Rocking scans, along the $Q_{x}$ direction and show that the width of the specular line is resolution limited undermining the absence of correlated roughness. Some years later, in the beginning of the 90's, the first GISANS experiment using neutrons was done showing a hexagonal alignment of CTA35ClBz, CTABr threadlike micelles at the solid-liquid boundary under Poiseuille flow [34]. Later GISANS was used to study the lamellar orientation of supported thin films of poly(styrene-b-butadiene). It turns out that in low molar mass samples the lamellae are parallel to the substrate surface, whereas they are perpendicular for high molar masses [32]. Some of the earlier OFFSpec and GISANS experiments are summarized in the short review by Dalgliesh [33] and Hamilton [34], respectively. A more recent review discusses the use of GISAXS and GISANS for the study of block copolymer thin films [35] and a paper by Lauter et al. evaluates the power of so called complete reflectometry experiments evaluating NR, OFFSpec and GISANS [36, 37]

The low absorption of neutrons can be used to probe not only the reflected but also transmitted (Laue 
scattering) intensity measuring below the samples horizon. This offers the opportunity to access a wide range of $Q$ values [38], e.g. for the study of highly aligned phospholipid membranes. Transmitting the neutron beam trough a quartz crystal and reflecting from water polymer-supported single lipid bilayers allows the study of model cell membranes [39]. From off-specular neutron scattering the in-plane height-height correlations of interfacial fluctuations of such a lipid bilayer can be quantified. It turns out that with decreasing temperature the polymer swells and the polymer supported lipid membrane deviates from its initially nearly planar structure with a decrease of the correlation length characteristic for capillary waves. Ultra-thin deuterated poly(methyl methacrylate) films may spinodally dewet on polystyrene substrates as a result of dispersive force driven instabilities consisting of thermally excited capillary waves. The length scale and growth rate of this instability was studied by NR and OFFSpec measurements and are consistent with the predictions of a linear theory of spinodal dewetting at a liquid/liquid interface [40]. The kinetics during the dewetting process of blend thin films deuterated polystyrene and poly vinyl methyl ether was studied by time-resolved specular and OFFSpec NR measurements. It is reported that the droplets formation on the micrometer length scale occurs in the late stage of dewetting [41].

As indicated above the self assembly of three block polymer micelles at solid interfaces is a good showcase for GIS from solid-liquid boundaries and a range of molecules has been investigated. The alignment of the crystallites at a surface was found somewhat similar to shear alignment in a combined GISANS and transmission SANS experiment [42]. In addition the texture of the self assembly [11] as well as the reconstruction of the structure after the cession of shear [45] strongly depends on the surface energy of the solid substrate. For micellar solutions showing a phase transition from cubic to hexagonal ordering a hysteresis in the transition temperature has been reported [20, 43, 44].

Some neutron reflectometers offer the possibility to measure from horizontal samples and liquid surfaces. Such a geometry can be used to study surfactant layers at the air-water interface, like the ones used for deposition in the Langmuir-Blodgett technique. Following along this line it was shown from NR profiles that for surface pressures below the collapse point of a low molecular weight polystyrene film only specular reflection is present, but in the collapse region, some OFFSpec develops, indicating the presence of surface texture on the micron length scale in the plane of the interface [46]. The temperature effect on the conformal roughness in aerosol-OT lamellar adsorbed at the air-water and liquid-solid interface was studied by $\mathrm{Li}$ et al. [47]. They show that with increasing temperature the OFFSpec scattering becomes enhanced close to the specular line, indicating fluctuations of the membrane layers with defined correlation length and amplitude. In a combined grazing incidence neutron scattering and AFM study Gliss et al. have investigated the size and orientation of phospholipid bilayers [48]. They show that in the coexistence region of gel and fluid phase the domains grow in number rather than in size with decreasing temperature. Similar to the air-liquid interfaces liquid-liquid interfaces can be studied and some early experiments using X-ray and neutron GIS methods are reviewed by Schlossman [49].

In the field of magnetism OFFSpec and GIS offers unique opportunities to study the formation of magnetic domains [26]. The incorporation of magnetic polystyrene coated nano-particles into a lamellar polystyrene-poly(butyl methacrylate) $\mathrm{P}(\mathrm{Sd}-\mathrm{b}-\mathrm{BMA}$ ) film was studied and it was shown that an increasing particle concentration in the polystyrene layers results in a thickness expansion and decreasing correlated roughness [50]. The self-assembly of magnetic particles at a silicon surface as well as at the air interface was studied by OFFSpec neutron scattering [51] and from polarized GISANS experiments combined with reflectometry the magnetization and assembly of magnetic particles on a silicon substrate was studied [52]. 


\section{Summary}

GIS methods become a more readily accessible scientific tool for the study of surfaces and interfaces. With more sophisticated instrumentation and data evaluation tools one can expect a considerable increase in the number of scientific studies resulting from this approach. In this article the method of GIS is discussed, the peculiarities of the scattering geometry are highlighted and some recent results are reviewed. The key features of GIS and OFFSpec scattering are the wide range of length scales, from sub-nm to about $100 \mu \mathrm{m}$, being accessible along the different scattering directions. In the case of neutrons the depth sensitivity is limited due to the low brilliance of the source and weak absorption in most materials. The efforts of developing GIS methods is not restricted to elastic scattering experiments. During the last years several attempts have been made to study the near surface dynamics by grazing incidence neutron spin echo (GINSES) experiments [53-57].

\section{References}

[1] G. Binnig, C. F. Quate, Ch. Gerber, Phys. Rev. Lett., 56, 930 (1986).

[2] R. Reichelt, Scanning Electron Microscopy, Science of microscopy (Springer, New York, NY, 2007), 133-272.

[3] http.//www.reflectometry.net/biblio/Neutron_reflectometer_bibliography_instrument_sort.pdf

[4] J. Daillant, A. Gibaud (X-ray and neutron reflectivity: Principles and applications, Springer, Lecture notes in Physics 770, 2009)

[5] M. Wolff, A. Magerl and H. Zabel, Euro. Phys. J. E, 16(2), 141 (2005).

[6] G.Wanka, H.Hoffmann, W.Ulbricht, Macromolecules 27, 4145 (1994).

[7] K. Mortensen, Polym. Adv. Technol. 12, 2 (2001).

[8] L.G. Parratt, Phys. Rev. 95, 359 (1954).

[9] H. Dosch, B. W. Batterman, D. C. Wack, Phys. Rev. Lett. 56, 1144 (1986).

[10] D. van der Grinten, M. Wolff, H. Zabel, A. Magerl, Meas. Sci. Technol. 19, 034016 (2008).

[11] M. Wolff, U. Scholz, R. Hock, A. Magerl and H. Zabel, Phys. Rev. Lett., 92, 255501 (2004).

[12] M. Ay, C. Schanzer, M. Wolff, J. Stahn, Nuclear Instr. and Methods in Phys. Res. A 562, 389 (2006).

[13] F. Adlmann, G. K. Palsson, J.-C. Bilheux, J. F. Ankner, P. Gutfreund, M. Kawecki, M. Wolff, J. Appl. Cryst. 49(6), 2091 (2016).

[14] Y. Yoneda, Phys. Rev. 131, 2010 (1963).

[15] V. Holy, T. Baumbach, Phys. Rev. B 49, 10668 (1994).

[16] M. Wolff, A. Magerl, H. Zabel, Langmuir (Letter) 25 (1), 64 (2009).

[17] F. Adlmann, G. K. Palsson, A. Korolkovas, J. Herbel, A. Korolkovas, B. Kitchen, A. Bliersbach,

B. P. Toperverg, W. van Herck, M. Wolff, J. Phys.: Cond. matter (submitted).

[18] M. Wolff, J. Herbel, F. Adlmann, A. J. C. Dennison, G. Liesche, P. Gutfreund, S. Rogers, J. Appl. Cryst. 47, 130 (2014).

[19] Müller-Buschbaum, G. Kaune, M. Haese-Seiller and J.-F. Moulin, J. Appl. Cryst. 47, 1228 (2014).

[20] N. Wolff, S. Gerth, P Gutfreund, M. Wolff, Soft Matter 10(42), 8420 (2014).

[21] S. K. Sinha, E. B. Sirota, S. Garoff, H. B. Stanley, Phys. Rev. B 38, 2297 (1988).

[22] George H. Vineyard, Phys. Rev. B 26, 4146 (1982).

[23] F. Pfeiffer, V. Leiner, P. Hoghoj, I. Anderson, Phys. Rev. Lett. 88, 055507 (2002).

[24] S. Jaksch, O. Holderer, M. Gvaramia, M. Ohl, M. Monkenbusch, H. Frielinghaus, Scientific Reports 7, 4417 (2017). 
[25] K. Theis-Bröhl, M. Wolff, A. Westphalen, H. Zabel, J. McCord, V. Höink, J. Schmalhorst, G. Reiss, T. Weis, D. Engel, A. Ehresmann, U. Rücker, B. P. Toperverg, Phys. Rev. B 73, 174408 (2006).

[26] Hartmut Zabel, Katharina Theis-Bröhl, B. P. Toperverg, Polarized Neutron Reflectivity and Scattering from Magnetic Nanostructures and Spintronic Materials in book: Handbook of Magnetism and Advanced Magnetic Materials, (John Wiley \& Sons, Ltd., 2007).

[27] http://bornagainproject.org/news

[28] M. Tolan, X-ray Scattering from Soft Matter Thin Films (Springer Tracts in Modern Physics vol. 148, Berlin, 1999).

[29] P. Müller-Buschbaum, Analytical and Bioanalytical Chemistry 376, 3 (2003).

[30] S. H. Anastaisiadis, T. P. Russel, S. K. Satija, C. F. Majkrzak, Phys. Rev. Lett. 62, 1852 (1989).

[31] W. A. Hamilton, P. D. Butler, S. M. Baker, G. S. Smith, John B. Hayter, L. J. Magid, and R. Pynn, Phys. Rev. Lett. 74, 335 (1995).

[32] P. Busch, D. Posselt, D.-M. Smilgies, M. Rauscher, C. M. Papadakis, Macromolecules 40, 630 (2007).

[33] R. Dalgliesh, Current Opinion in Colloid \& Interface Science 7, 244 (2002).

[34] W. A. Hamilton, Current Opinion in Colloid \& Interface Science 9, 390 (2005).

[35] Müller-Buschbaum, European Polymer Journal 81, 470 (2016).

[36] H.J.C. Lauter, V. Lauter, B.P. Toperverg, Polymer Science: A Comprehensive Reference 2, 411 (2012).

[37] V. Lauter, H.J.C. Lauter, A. G. Glavic, B.P. Toperverg, Reflectivity, Off-Specular Scattering, and GISANS Neutrons, in book: Reference Module in Materials Science and Materials Engineering, (Oxford: Elsevier, Editors: Saleem Hashmi, pp.1-27 (2016).

[38] C. Münster, T. Salditt, M. Vogel, R. Siebrecht, J. Peisl, Europhys. Lett. 46, 486 (1999).

[39] M. S. Jablin, M. Zhernenkov, B. P. Toperverg, M. Dubey, H. L. Smith, A. Vidyasagar, R. Toomey, A. J. Hurd, J. Majewski, Phys. Rev. Lett. 106, 138101 (2011).

[40] M. Sferrazza, M. Heppenstall-Butler, R. Cubitt, D. Bucknall, J. Webster, R. A. L. Jones, Phys. Rev. Lett. 81, 5173 (2009).

[41] H. Ogawa, T. Kanaya, K. Nishida, G. Matsuba, J. P. Majewski, E. Watkins, J. Phys. Chem. 131, 104907 (1994).

[42] M. Wolff, A. Magerl, H. Zabel, Thin solid films 515, 5724 (2007).

[43] M. Wolff, A. Magerl, H. Zabel, J. Phys.: Cond. Matter 17, S3645 (2005).

[44] M. Walz, M. Wolff, N. Voss, A. Magerl, H. Zabel, Langmuir 26, 14391 (2010).

[45] M. Wolff, R. Steitz, P. Gutfreund, N. Voss, S. Gerth, M. Walz, A. Magerl, H. Zabel, Langmuir (Letter) 24, 11331 (2008).

[46] P. M. Saville, I. R. Gentle, J. W. White, J. Penfold, J. R. P. Webster, J. Phys. Chem. 98, 5935 (1994).

[47] Z.X. Li, J. R. Lu, R. K. Thomas, A. Weller, J. Penfold, R. P. Webster, D. S. Sivia, A. R. Rennie, Langmuir 17, 5858 (2001).

[48] C. Gliss, H. Clausen-Schaumann, R. Günther, S. Odenbach, O. Randl, T.M. Bayerl, Biophysical Journal 742443 (1998).

[49] M. L. Schlossman, Current Opinion in Colloid \& Interface Science 7, 235 (2002).

[50] V. Lauter-Pasyuk, H. J. Lauter, G. P. Gordeev, P. Muüller-Buschbaum, B. P. Toperverg, M. Jernenkov, W. Petry, Langmuir 19, 7783 (2003). 
[51] A. Vorobiev, G. Gordeev, W. Donner, H. Dosch, B. Nickel, B.P. Toperverg, Physica B 297194 (2001).

[52] K. Theis-Bröhl, M. Wolff, I. Ennen, C. Dewhurst, A. Hütten, B. P. Toperverg, Phys. Rev. B 78, 134426 (2008).

[53] H.Frielinghaus, O.Holderer, F.Lipfert, M.Monkenbusch, N.Arend, D.Richter, Nuclear Inst. and Methods in Physics Research, A 686, 71 (2012).

[54] F. Lipfert, H. Frielinghaus, O. Holderer, S. Mattauch, M. Monkenbusch, N. Arend, and D. Richter, Phys. Rev. E 89, 042303 (2014).

[55] K. Gawlitza, O. Ivanova, A. Radulescu, O. Holderer, R. von Klitzing, S. Wellert, Macromolecules 48(16), 5807 (2015).

[56] S. Jaksch, O. Holderer, M. Gvaramia, M. Ohl, M. Monkenbusch, H. Frielinghaus, Sci Rep. 7, 4417 (2017).

[57] H. Frielinghaus, M. Gvaramia, G. Mangiapia, S. Jaksch, M. Ganeva, A. Koutsioubas, S. Mattauch, M. Ohl, M. Monkenbusch, O. Holderer, Nuclear Inst. and Methods in Physics Research A 871, 72 (2017). 